\title{
钒氧化物电极材料晶格呼吸现象原位探测及其反应机制研究
}

\author{
张国涁 ${ }^{\dagger}$ 熊腾飞门潘雪雷 ${ }^{\dagger}$ 晏梦雨 韩春华麦立强* \\ (材料复合新技术国家重点实验室 武汉理工大学材料科学与工程学院 武汉 430070)
}

\begin{abstract}
摘要 作为锂离子电池正极材料, 层状钒氧化物具有优异的物理特性和良好的储锂性能, 因此被广泛研究与应用于工 业生产与日常生活的各个方面. 然而, 在脱嵌锂的过程中, $\mathrm{V}_{2} \mathrm{O}_{5}$ 凝胶等层状钒氧化物的层状结构存在 “晶格呼吸” 现象. 这种现象导致了电极材料的针化, 并进一步导致电池容量快速衰减. 采用了原位 X 射线衍射(XRD)来研究一种层状钒 氧化物 $\left(\mathrm{VO}_{x}\right)$ 在充放电过程中的 “晶格呼吸” 现象, 揭示了其独特的相变过程. 在充放电过程中, 原位 XRD 对应的二维 衍射图显示出三个不同的阶段，分别对应三个固溶反应. 放电过程中，三个阶段的衍射峰都向高角度偏移，表明在锂 离子嵌入过程中, $\mathrm{VO}_{x}$ 的层间距存在持续收缩过程. (001)层间距大小随充放电过程的变化图进一步证明了这三个过程的 不连续性. 这些发现揭示了这类材料在电极反应过程中的晶体结构变化规律以及造成其容量衰减的原因.
\end{abstract}

关键词 锂离子电池; 正极材料; 层状钒氧化物; 原位; 相变

\section{In Situ Observation and Mechanism Investigation of Lattice Breathing in Vanadium Oxide Cathode}

\author{
Zhang, Guobin ${ }^{\dagger} \quad$ Xiong, Tengfei ${ }^{\dagger} \quad$ Pan, Xuelei $^{\dagger} \quad$ Yan, Mengyu \\ Han, Chunhua Mai, Liqiang*
}

(State Key Laboratory of Advanced Technology for Materials Synthesis and Processing, School of Materials Science and Engineering, Wuhan University of Technology, Wuhan 430070)

\begin{abstract}
As cathode materials in lithium-ion batteries, layered vanadium oxides have been extensively studied and used in many aspects varying from industrial production to our daily life, due to their excellent physical property and gorgeous lithium storage performance. During lithiation/delithiation, layered vanadium oxides such as $\mathrm{V}_{2} \mathrm{O}_{5}$ xerogel (with a bilayer structure), undergoes "lattice breathing" which leads to the deactivation of electrode materials and fast capacity fading, which limits its large-scale application. In this work, $\mathrm{VO}_{x}$ is used as the cathode material of lithium-ion batteries to study the "lattice breathing" phenomenon. The phase evolution has been observed and studied via in situ method. The X-ray diffraction (XRD) patterns show typical (001) diffraction peaks characteristic of vanadium oxide xerogel structure and also confirm the good crystallinity. This compound with crystal parameters of $a=4.56 \AA, b=14.87 \AA, c=12.38 \AA, \alpha=117.26^{\circ}, \beta=96.02^{\circ}, \gamma=$ $81.86^{\circ}$, forms a triclinic structure. Results of scanning electron microscope (SEM) and transmission electron microscope (TEM) further verify the layered structure of $\mathrm{VO}_{x}$. The thermo gravimetric analysis (TGA) at air and nitrogen atmosphere shows that the carbon content of the sample is about $2.4 \mathrm{wt} \%$ and the water content is about $2.1 \%$. As lithium-ion battery cathode the initial discharge capacity of the compound is about $136 \mathrm{~mA} \cdot \mathrm{h} / \mathrm{g}$ at a current density of $100 \mathrm{~mA} / \mathrm{g}$, with a capacity retention of $92.6 \%$ after 50 cycles. To study the lithium storage mechanism of $\mathrm{VO}_{x}$, electrochemical discharge/charge processes are further investigated by in situ XRD. It is found that the lattice plane diffraction displays three different stages linked during the insertion and deinsertion of lithium ions, indicating three solid solution reactions. During discharge process, the three diffraction changes show continuous shifts to higher diffraction angles, demonstrating three different continuous contraction processes with the insertion of lithium ions. Nevertheless, the evolution of the (001) peak is swift during the beginning and the end of discharge, in contrast to the slow deviation of the intermediate process. In the whole process, the diffraction pattern displays periodic changes, confirming the reversibility of the reaction process. The corresponding calculations of $d_{001}$ during the discharge/charge process prove the notable discontinuity between these three stages. In addition, cycling experiments conducted at the higher and the lower temperature indicate that the electrochemical performance of this compound is highly sensitive to temperature.

Keywords lithium-ion battery; cathode material; layered vanadium oxides; in situ; phase transition
\end{abstract}

*E-mail: mlq518@whut.edu.cn

Received March 3, 2016; published June 6, 2016.

Supporting information for this article is available free of charge via the Internet at http://sioc-journal.cn.

Project supported by the International Science \& Technology Cooperation Program of China (No. 2013DFA50840), the National Science Fund for Distinguished Young Scholars (No. 51425204) and the National Basic Research Program of China (Nos. 2013CB934103, 2012CB933003).

项目受国家国际科技合作计划(No. 2013DFA50840)、国家杰出青年科学基金(No. 51425204)和国家 973 计划(Nos. 2013CB934103, 2012CB933003)资助.

These authors contributed equally to this work. 


\section{1 引言}

为充分利用可再生能源, 并使电力供应实现削峰填 谷的目标, 发展大规模储能系统是十分有必要的 ${ }^{[1-3]}$. 可行的大规模储能系统应满足能量密度大、使用寿命 长、安全性高、成本低等基本要求. 在众多的储能技术 中, 可充电二次电池是最具前景的化学储能方法之 一 ${ }^{[4 \sim 6]}$. 在二次电池中, 锂离子电池由于其较高的比容 量及能量转换效率而被广泛研究与应用 ${ }^{[7,8]}$. 在锂离子 电池的正极材料中, 过渡金属氧化物因具有比容量高、 原材料丰富等优点而引起研究者的关注 ${ }^{[9 \sim 17]}$. 其中, 层 状的钒氧化物如 $\mathrm{V}_{2} \mathrm{O}_{5}, \mathrm{~V}_{6} \mathrm{O}_{13}, \mathrm{VO}_{2}(\mathrm{~B})$ 等表现出较好的储 锂性能 ${ }^{[18 ~ 20]}$. 为进一步优化层状钒氧化物的电化学性 能, 许多研究者致力于扩大其层间距 ${ }^{[21 ~ 23]}$. 其中, $\mathrm{V}_{2} \mathrm{O}_{5}$ 凝胶的层间距较大, 可达 $14.0 \AA$ 以上, 超过了三斜晶系 $\mathrm{V}_{2} \mathrm{O}_{5}$ 的层间距, 是较为理想的锂离子电池正极材 料 ${ }^{[18,24,25]}$. 然而, 正是由于其层间距较大, $\mathrm{V}_{2} \mathrm{O}_{5}$ 凝胶的 双层结构在离子嵌入时收缩明显, 在离子脱出时膨胀严 重, 导致充放电过程中结构不稳定 ${ }^{[26,27]}$. 这种 “晶格呼 吸” 现象导致了电极的钝化以及随之而来的容量衰 减 ${ }^{[28,29]}$. 为了解决这个问题, 必需首先将 “晶格呼吸” 对应的相变过程研究透彻. 最近, 我们课题组报道了一 种层状钒氧化物 $\left(\mathrm{VO}_{x}\right)$, 并发现 $\mathrm{VO}_{x}$ 在离子脱嵌过程中 伴随明显的 “晶格呼吸” 现象 ${ }^{[26]}$. 然而, $\mathrm{VO}_{x}$ 在充放电过 程中的反应机理并没有被仔细探讨. 本工作以同样的材 料 $\left(\mathrm{VO}_{x}\right)$ 作为锂离子电池的正极材料, 采用原位 XRD 技 术研究了在锂离子脱嵌过程中 $\mathrm{VO}_{x}$ 的相变机理, 发现了 三个可逆的固溶反应. 这一研究结果为进一步研究层状 钒氧化物的电化学性能提高其循环稳定性以及改性提 供了理论依据.

\section{2 结果与讨论}

\section{1 样品的物相和形貌分析}

合成的样品首先采用 XRD 进行粉末衍射测试. XRD 图谱(图 1d)显示出钒氧化物凝胶结构的(001)型衍

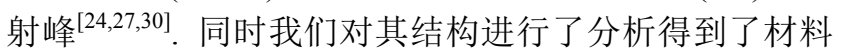
的结构参数为: $a=4.56 \AA, b=14.87 \AA, c=12.38 \AA, \alpha=$ $117.26^{\circ}, \beta=96.02^{\circ}, \gamma=81.86^{\circ}$, 属于三斜晶系. 进而采用 扫描电子显微镜和透射电子显微镜来观测样品的形貌 和结构. 如图 $1 \mathrm{a}, 1 \mathrm{~b}$ 所示, 所合成的样品具有典型的层 状结构. 高分辨透射电子显微镜结构表明材料具有很好 的结晶性. 根据空气中测得的热重分析(TGA)曲线(图 2) 和氮气中的 TGA 曲线(图 S1) 可以看出, 在 $400{ }^{\circ} \mathrm{C}$ 之前, 由于材料中的水不断被空气中的氧气消耗, 材料的质量 呈递减趋势. 材料的质量呈递减趋势. $400{ }^{\circ} \mathrm{C}$ 后, 材料 中的钒元素被氧化, 因此质量有所增加. 由此可以得知 材料中碳的质量百分含量约为 $2.4 \%$, 水含量约为 $2.1 \%$. 在提高材料导电性的同时, 如此低的碳含量对材料整体
的电化学性能不会产生很大影响. 同时综合 X射线光电 子能谱 (XPS) 分析 (图 S2) 可得该材料具体化学式为 $\mathrm{VO}_{2.4} \bullet 0.2 \mathrm{H}_{2} \mathrm{O}$.
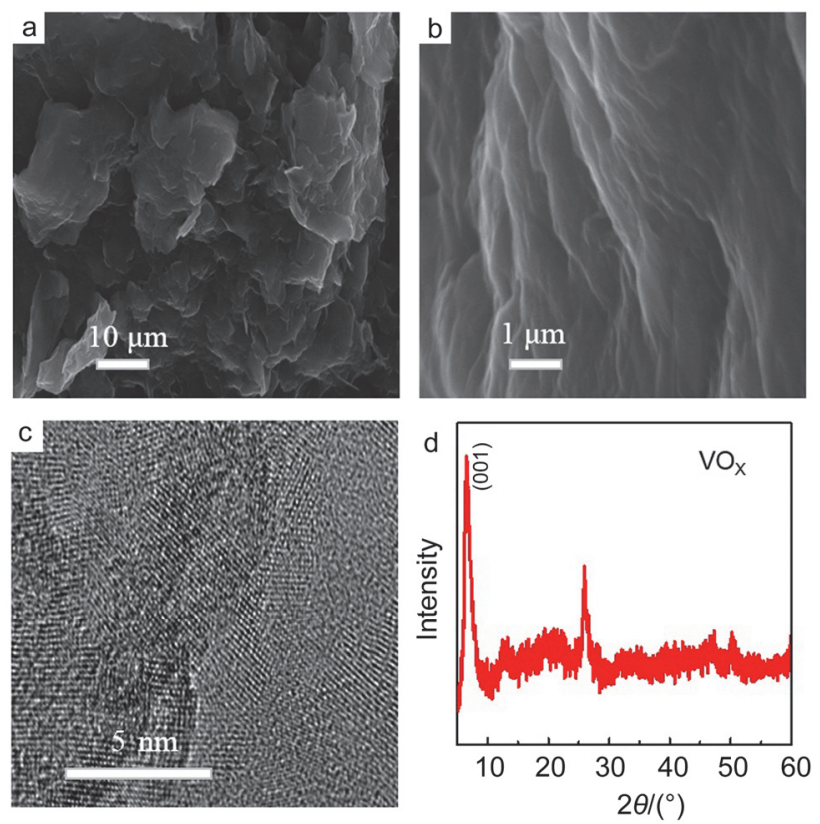

图 $1 \mathrm{VO}_{x}$ 的低倍 SEM 照片(a)、高倍 SEM 照片(b)、TEM 照片(c)及 XRD 图谱(d)

Figure 1 SEM images of low- (a) and high- (b) magnification and TEM image (c) and XRD pattern (d) of $\mathrm{VO}_{x}$

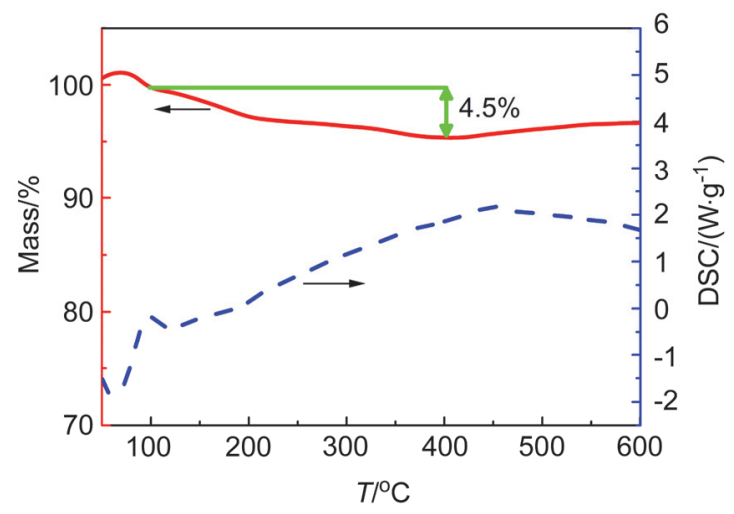

图 $2 \mathrm{VO}_{x}$ 对应空气中的热重曲线

Figure 2 TG-DSC curves of $\mathrm{VO}_{x}$ at air

\section{2 电化学性能测试}

图 3 为组装得到的 $\mathrm{VO}_{x}$ 电极前 50 次的恒流充放电曲 线图, 其充放电电压区间为 $1.5 \sim 4 \mathrm{~V}$, 充放电电流密度为 $100 \mathrm{~mA} / \mathrm{g}$. 由充放电曲线可知, 首圈放电容量达 136 $\mathrm{mA} \cdot \mathrm{h} / \mathrm{g}$, 经过 50 圈的充放电循环后, $\mathrm{VO}_{x}$ 的容量保持率 高达 $92.6 \%$, 且库伦效率始终在 $97 \%$ 以上, 说明在后续的 循环过程中 $\mathrm{VO}_{x}$ 电极具有较强的稳定性和可逆性.

\section{3 电化学机理分析}

为研究 $\mathrm{VO}_{x}$ 的储锂机制，在充放电的同时采用了原 位 XRD 技术进行材料物相变化实时监测. 如图 4 所示, 


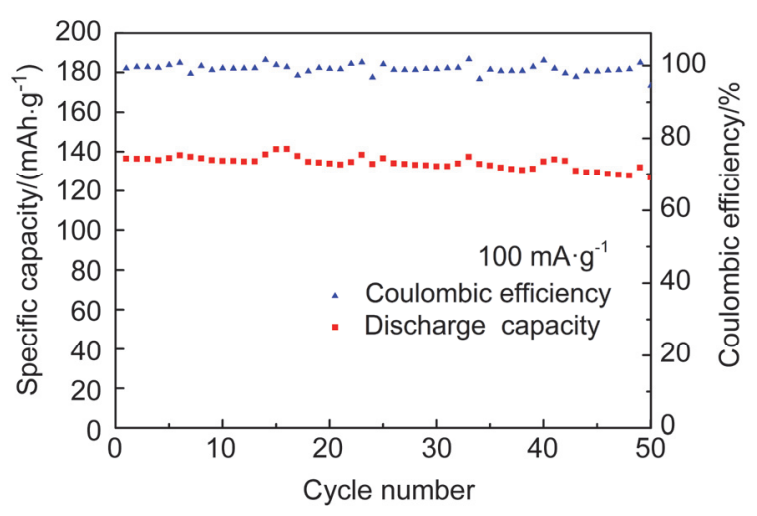

图 $3 \mathrm{VO}_{x}$ 电极的循环性能图

Figure 3 Cycling performance of $\mathrm{VO}_{x}$ electrode

衍射峰的轮廓线清楚地显示了在电流密度为 $100 \mathrm{~mA} / \mathrm{g}$ 时充放电过程中的相变过程. 在充放电过程中, 大多数 衍射峰变化不明显, 只有少量衍射峰对锂离子脱嵌过程 有明显反应. 其中 $(001)$ 晶面的衍射峰对锂离子的脱嵌 十分敏感, 在整个充放电过程中变化明显 ${ }^{[31]}$, 因此本实 验选择(001) 衍射峰来原位监测相变过程 ${ }^{[32,33]}$. 整个放 电过程分为三个阶段. 在阶段 I, 随着最初少量锂离子 的嵌入, (001)衍射峰迅速向高衍射角偏移, 表明此过程 中 $\mathrm{VO}_{x}$ 的层间距迅速减小. 这种层结构紧缩现象主要是 由于带正电的锂离子与带负电的氧原子之间存在强烈 的静电吸引力. 在(阶段 II)电压平台 $2.55 \mathrm{~V}$ 处, 随着更 多锂离子的嵌入, (001)衍射峰依旧向高角度偏移, 但偏 移的速度十分缓慢. 在这一过程中, 一方面由于层间的 锂离子数量已经较多, 锂离子间的相互排斥力增大, 另 一方面, $\mathrm{VO}_{x}$ 靠近的两层之间排斥力也在不断增大, 导 致 $\mathrm{VO}_{x}$ 双层之间的继续靠近受到较大阻碍 ${ }^{[34,35]}$. 在(阶 段 III)电压平台 $2.15 \mathrm{~V}$ 处, 随着放电继续进行, (001) 衍 射峰再次以较大速率偏向高角度, 放电结束后对应 $2 \theta$ 值为 $9.4^{\circ}$. 这一过程除偏移速度与阶段 II 明显不同外, 其衍射强度也明显减弱, 表明晶体结构的部分破坏. 因 此, 合理的解释是, 在锂离子嵌入的末期, 虽然各种斥 力在不断增大, 但锂离子与氧原子之间吸引力再次在层 结构变化中占据绝对优势. 在随后的过程中, (001)衍射 峰随着充电的进行显示出与放电过程完全一致的衍射 图样, 表明了此电化学过程的高度可逆性. 从整个充放 电过程来看, (001)衍射峰有着周期性的变化规律, 显示 出 $\mathrm{VO}_{x}$ 层状框架在锂离子脱嵌过程中的稳定性 ${ }^{[35,36]}$. 通 过分析其最强峰在电化学反应过程中的演变发现, 其衍 射峰所对应晶面间距 $d$ 的变化如图 5 所示, 其变化范围 为 $9.5 \sim 11.5 \AA$, 对应的变化率为 $10 \%$. 而 $d$ 值所对应的 突变点即为反应过程中平台的转变点, 反映出晶体结构 在此条件下的响应情况. 可以看出其主要突变有两个, 第一个为初始过程到放电开始时的过程, 第二个为 2.2 $\mathrm{V}$ 时平台骤变情况. $d$ 值的对称可逆变化表明充放电过 程为可逆相变反应. 图 6 为电流密度为 $500 \mathrm{~mA} / \mathrm{g}$ 下的高
低温循环测试 $\left(-20{ }^{\circ} \mathrm{C}\right.$ 和 $\left.60{ }^{\circ} \mathrm{C}\right)$. 从低温下的循环曲线 可以看出, 由于较低的离子传导性及电导率, 低温下的 循环比容量普遍低于室温 ${ }^{[37,38]}$. 另外, 低温下的电压平 台不如室温下明显. 与低温条件相反，高温下较高的反 应活性使电极材料的比容量提高、循环性能下降, 但其 电压平台几乎消失 ${ }^{[39]}$. 由此可以看出 $\mathrm{VO}_{x}$ 的电化学性能 对温度较为敏感.

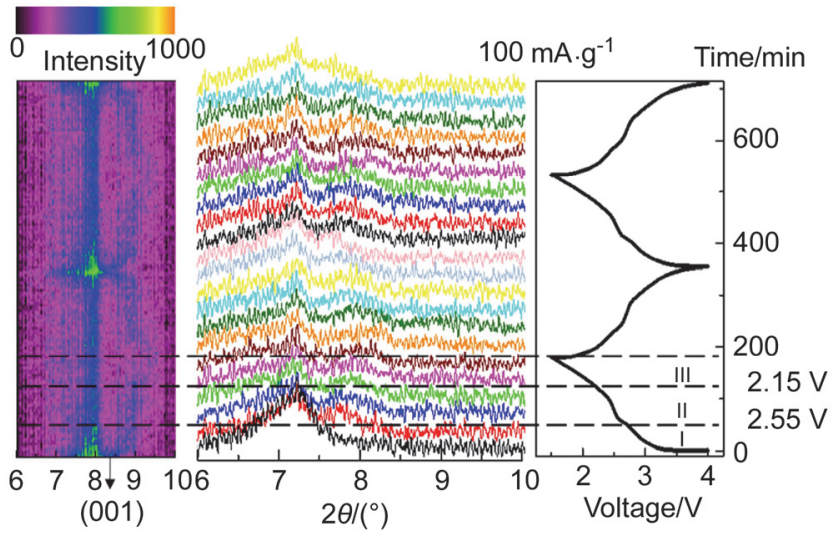

图 4 恒流充放电时 $\mathrm{VO}_{x}$ 的原位 XRD 图谱

Figure 4 In situ XRD patterns collected during galvanostatic discharge/charge process

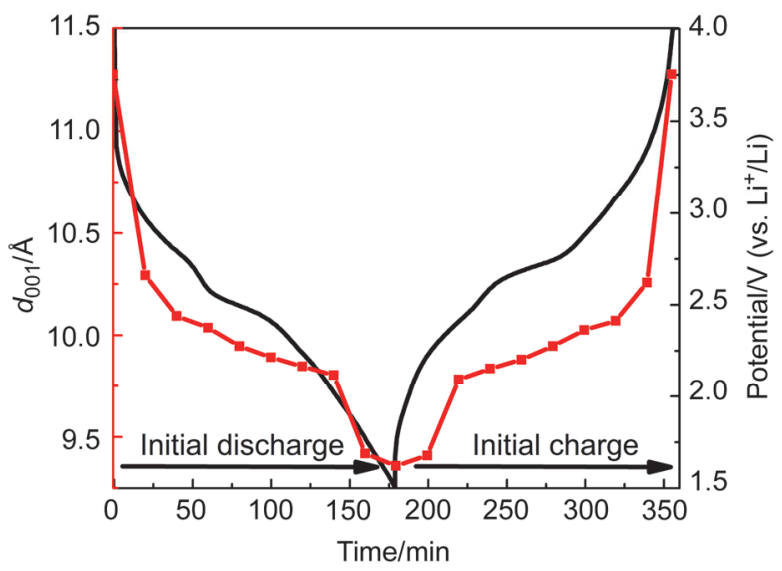

图 5 (001)层间距大小随充放电过程的变化

Figure 5 Evolution of $d_{001}$ during the discharge/charge process

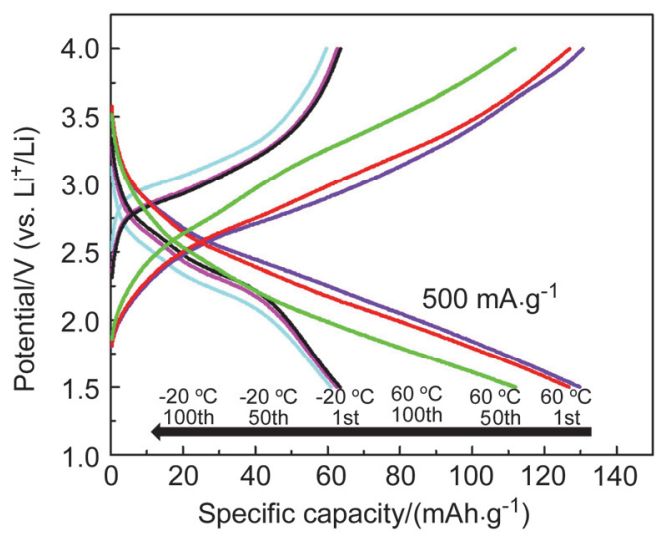

图 $6-20$ 及 $60{ }^{\circ} \mathrm{C}$ 时分别对应的充放电曲线

Figure 6 Charge/discharge curves for temperatures at -20 and $60{ }^{\circ} \mathrm{C}$ 


\section{3 结论}

采用原位 XRD 研究了锂离子脱嵌过程中 $\mathrm{VO}_{x}$ $\left(\mathrm{VO}_{2.4} \cdot 0.2 \mathrm{H}_{2} \mathrm{O}\right)$ 的相演变过程. 在大多数文献的报道中, 当锂离子嵌入层状化合物时, 化合物的双层结构会膨胀 导致层间距变大. 但对于 $\mathrm{VO}_{x}$ 嵌锂时, 其层间距是连续 的变小过程. 原位 XRD 表征将嵌锂过程分为三个阶段. 在初始放电阶段, 由于锂离子与氧原子之间强烈的静电 吸引力, $\mathrm{VO}_{x}$ 双层结构快速压缩, (001) 衍射峰迅速向高 衍射角偏移. 此后的阶段 II 中, 一方面锂离子间的相互 排斥力增大, 另一方面 $\mathrm{VO}_{x}$ 层间的排斥力也在不断增 大. 因此 $\mathrm{VO}_{x}$ 双层之间的继续靠近受到较大阻碍, (001) 衍射峰依旧向高角度偏移, 但偏移的速度十分缓慢. 在 最后的阶段 III, 虽然各种斥力在不断增大, 但锂离子与 氧原子之间吸引力再次在层结构变化中占据绝对优势, (001) 衍射峰再次以较大速率偏向高角度. 随后的充电 过程中, (001) 对应的衍射图样的变化与放电过程完全 一致, 表明了此电化学过程的高度可逆性. 从整个充放 电过程来看, (001)衍射峰有着周期性的变化规律, 显示 出 $\mathrm{VO}_{x}$ 层状框架在锂离子脱嵌过程中的稳定性. 这样的 发现揭示了锂离子电池电极材料中特有的一种晶格呼 吸现象, 这种钝化材料的反应过程被全面的研究, 发现 在这个相演变中晶体结构的变化率达到了 $10 \%$, 这样大 的连续的变化率是先前所没有发现的. 并且这样的演变 揭示了我们在后期改性过程中要利用一些例子来稳定 材料的晶格, 实现抑制这种变化的发展扩大.

\section{4 实验部分}

\section{1 材料制备}

$\mathrm{VO}_{x}$ 采用文献[26]中报道的方法进行合成. 首先, 将 $0.3 \mathrm{mmol}$ 乙酰丙酮(分析纯)溶解于 $38.5 \mathrm{~mL}$ 去离子水 中, 搅拌均匀. 继续加入 $0.064 \mathrm{~mol} / \mathrm{L}$ 的 $\mathrm{V}_{2} \mathrm{O}_{5}$ 溶胶 15.5 $\mathrm{mL}$ 并摚拌 $1 \mathrm{~h}$. 搅拌完后, 将得到的均一液体置于 100 $\mathrm{mL}$ 反应釜中, 在 $180{ }^{\circ} \mathrm{C}$ 下保温 $48 \mathrm{~h}$. 反应釜冷却至室 温后, 将得到的沉淀用去离子水和乙醇各洗涤三次, 之 后分散在去离子水中并进行冷冻干燥处理. 最后, 将冷 冻干燥得到的样品在 $150{ }^{\circ} \mathrm{C}$ 下真空干燥 $5 \mathrm{~h}$ 即得样品.

\section{2 结构和形貌表征}

采用布鲁克 D8 Discover 型 X 射线衍射仪 $(\mathrm{Cu}$ 靶)进 行粉末衍射实验, 以收集相关晶体学信息, 对应的 $2 \theta$ 角 范围为 $10^{\circ} \sim 80^{\circ}$. SEM 图采用 JEOL-7100F 式扫描电镜 收集, TEM 图采用 JEM-2100F 式透射电镜收集, 对应的 EDS 图采用 Oxford IE250 系统获得. 在界气氛为空气的 条件下, 热重一差示扫描分析(TG-DSC analyses)分别采 用 VG MultiLab 2000 及 STA-449F3 系统获得, 对应的 升温速率为 $5{ }^{\circ} \mathrm{C} / \mathrm{min}$. 采用 2016 型纽扣电池, 在充满纯 氩气的手套箱中进行组装. 以锂片为负极, 浓度为 1 $\mathrm{mol} / \mathrm{L}$ 的 $\mathrm{LiPF}_{6}$ 为电解液, 其中 $\mathrm{LiPF}_{6}$ 溶于体积比为 $1: 1$
的碳酸乙烯酯(EC), 碳酸二甲酯(DMC)混合液. 正极含 质量分数为 $70 \%$ 的活性材料, $20 \%$ 的乙炔黑以及 $10 \%$ 的 聚偏氟乙烯(PVDF). 恒流充放电测试采用多通道电池 测试系统(LAND CT2001A) 实现，电压区间为 1.5 4.0 V.

\section{5 致谢}

感谢武汉理工大学分析测试中心方德老师在分析 测试上给予本工作的支持.

\section{References}

[1] Larcher, D.; Tarascon, J. M. Nat. Chem. 2015, 7, 19.

[2] Pan, H. L.; Hu, Y. S.; Chen, L. Q. Energy Environ. Sci. 2013, 6, 2338.

[3] Zhang, Q. F.; Uchaker, E.; Candelaria, S. L.; Cao, G. Z. ChemInform 2013, 44, 3127.

[4] An, T. C; Wang, Y. H.; Tang, J.; Wang, Y.; Zhang, L. J.; Zheng, G. F. J. Colloid Interface Sci. 2015, 445, 320.

[5] Kim, H.; Hong, J. H.; Park, K. Y.; Kim, H.; Kim, S. W.; Kang, K. Chem. Rev. 2014, 114, 11788.

[6] Yang, C. P.; Yin, Y. X.; Ye, H.; Jiang, K. C.; Zhang, J.; Guo, Y. G. ACS Appl. Mater. Interfaces 2014, 6, 8789.

[7] Lyu, Z. Y.; Feng, R.; Zhao, J.; Fan, H.; Xu, D.; Wu, Q.; Yang, L. J.; Chen, Q.; Wang, X. Z.; Hu, Z. Acta Chim. Sinica 2015, 73, 1013. (吕之阳, 冯瑞, 赵进, 范豪, 徐丹, 吴强, 杨立军, 陈强, 王喜章, 胡征, 化学学报, 2015, 73, 1013.)

[8] Feng, R.; Wang, L. W.; Lyu, Z. Y.; Wu, Q.; Yang, L. J.; Wang, X. Z.; Hu, Z. Acta Chim. Sinica 2014, 72, 653. (冯瑞, 王立伟, 吴强, 吕 之阳, 杨立军, 王喜章, 胡征, 化学学报, 2014, 72, 653.)

[9] Armand, M.; Tarascon, J. M. Nature 2008, 451, 652.

[10] Armstrong, M. J.; O’Dwyer, C.; Macklin, W. J.; Holmes, J. D. Nano Res. 2014, 7, 1.

[11] Han, M. H.; Gonzalo, E.; Singh, G.; Rojo, T. Energy Environ. Sci. 2015, 8,81 .

[12] Lukatskaya, M. R.; Mashtalir, O.; Ren, C. E.; Dall'Agnese, Y.; Rozier, P.; Taberna, P. L.; Naguib, M.; Simon, P.; Barsoum, M. W.; Gogotsi, Y. Science 2013, 341, 1502.

[13] Naguib, M.; Gogotsi, Y. Acc. Chem. Res. 2014, 48, 128.

[14] Naguib, M.; Mochalin, V. N.; Barsoum, M. W.; Gogotsi, Y. $A d v$. Mater. 2014, 26, 992.

[15] Poizot, P.; Laruelle, S.; Grugeon, S.; Dupont, L.; Tarascon, J. M. Nature 2000, 407, 496.

[16] Reddy, M. V.; Subba Rao, G. V.; Chowdari, B. V. R. Chem. Rev. 2013, 113, 5364.

[17] Wei, Q. L.; Tan, S. S.; Liu, X. Y.; Yan, M. Y.; Wang, F. C.; Li, Q. D.; An, Q. Y.; Sun, R. M.; Zhao, K. N.; Wu, H. A. Adv. Funct. Mater. 2015, 25, 1773 .

[18] Chernova, N. A.; Roppolo, M.; Dillon, A. C.; Whittingham, M. S. J. Mater. Chem. 2009, 19, 2526.

[19] Dai, L.; Gao, Y. F.; Cao, C. X.; Chen, Z.; Luo, H. J.; Kanehira, M.; Jin, J.; Liu, Y. RSC $A d v . \mathbf{2 0 1 2}, 2,5265$.

[20] Wang, C. Q.; Liu, X. L.; Shao, J.; Xiong, W. M.; Ma, W. J.; Zheng, Y. RSC Adv. 2014, 4, 64021.

[21] Murugan, A. V.; Kale, B. B.; Kwon, C. W.; Campet, G.; Vijayamohanan, K. J. Mater. Chem. 2001, 11, 2470.

[22] Wang, Y.; Cao, G. Z. Chem. Mater. 2006, 18, 2787.

[23] Wang, Y.; Takahashi, K.; Lee, K. H.; Cao, G. Z. Adv. Funct. Mater. 2006, 16, 1133.

[24] Sathiya, M.; Prakash, A. S.; Ramesha, K.; Tarascon, J. M.; Shukla, A. K. J. Am. Chem. Soc. 2011, 133, 16291.

[25] Whittingham, M. S. Chem. Rev. 2004, 104, 4271.

[26] Wei, Q. L.; Jiang, Z. Y.; Tan, S. S.; Li, Q. D.; Huang, L.; Yan, M. Y.; Zhou, L.; An, Q. Y.; Mai, L. Q. ACS Appl. Mater. Interfaces 2015, 7, 18211.

[27] Wei, Q. L.; Liu, J.; Feng, W.; Sheng, J. Z.; Tian, X. C.; He, L.; An, Q. Y.; Mai, L. Q. J. Mater. Chem. A 2015, 3, 8070.

[28] Zhao, Y. L.; Han, C. H.; Yang, J. W.; Su, J.; Xu, X. M.; Li, S.; Xu, L.; Fang, R. P.; Jiang, H.; Zou, X. D. Nano Lett. 2015, 15, 2180.

[29] Zhou, Y. N.; Ma, J.; Hu, E. Y.; Yu, X. Q.; Gu, L.; Nam, K. W.; Chen, 
L. Q.; Wang, Z. X.; Yang, X. Q. Nat. Commun. 2013, 5, 5381.

[30] Liu, Q.; Li, Z. F.; Liu, Y. D.; Zhang, H. Y.; Ren, Y.; Sun, C. J.; Lu, W. Q.; Zhou, Y.; Stanciu, L.; Stach, E. A.; Xie, J. Nat. Commun. 2015, 6,6127 .

[31] Dong, Y. F.; Xu, X. M.; Li, S.; Han, C. H.; Zhao, K. N.; Zhang, L.; Niu, C. J.; Huang, Z.; Mai, L. Q. Nano Energy 2015, 15, 145.

[32] Berthelot, R.; Carlier, D.; Delmas, C. Nat. Mater. 2011, 10, 74.

[33] Liu, H.; Strobridge, F. C.; Borkiewicz, O. J.; Wiaderek, K. M.; Chapman, K. W.; Chupas, P. J.; Grey, C. P. Science 2014, 344, 1451.

[34] Wu, D.; Li, X.; Xu, B.; Twu, N.; Liu, L.; Ceder, G. Energy Environ. Sci. 2015, 8, 195.
[35] Yue, J. L.; Zhou, Y. N.; Shi, S. Q.; Shadike, Z.; Huang, X. Q.; Luo, J.; Yang, Z. Z.; Li, H.; Gu, L.; Yang, X. Q.; Fu, Z. W. Sci. Rep. 2014, 5,8810 .

[36] Li, Q. D.; Wei, Q. L.; Sheng, J. Z.; Yan, M. Y.; Zhou, L.; Luo, W.; Sun, R. M.; Mai, L. Q. Adv. Sci. 2015, 2, 1500284.

[37] Wu, X. L.; Guo, Y. G.; Su, J.; Xiong, J. W.; Zhang, Y. L.; Wan, L. J. Adv. Energy Mater. 2013, 3, 1155 .

[38] Liao, X. Z.; Ma, Z. F.; Gong, Q.; He, Y. S.; Pei, L.; Zeng, L. Electrochem. Commun. 2008, 10, 691.

[39] Yang, S.; Gong, Y.; Liu, Z.; Zhan, L.; Hashim, D. P.; Ma, L.; Vajtai, R.; Ajayan, P. M. Nano Lett. 2013, 13, 1596.

(Zhao, C.) 